\title{
Uso de harina de hojas de morera (Morus alba) en la alimentación de codornices en postura (Coturnix coturnix japonica)
}

\section{Use of leaf meal of Morus alba in feeding of laying quail (Coturnix coturnix}

japonica)

\author{
Bermúdez Rocio ${ }^{1}$ y Roa Vega María Ligia ${ }^{2}$ \\ ${ }^{1}$ Medico Veterinario Zootecnista, Universidad de los Llanos y \\ ${ }^{2}$ Zootecnista MSc, Docente, Escuela de ciencias Animales, Universidad de los \\ Llanos, Villavicencio, Colombia

\section{mroa@unillanos.edu.co}

Recibido 15 de Mayo 2013, Aceptado 15 de Julio 2013

\section{RESUMEN}

Este trabajo se realizó con el objetivo de evaluar el comportamiento productivo nutricional y económico, de codornices (Coturnix coturnix japonica) de la semana 2 a 23 de postura, utilizando harina de hojas de morera (Morus alba) en tres niveles de reemplazo del concentrado comercial, Fueron utilizadas 128 aves de 52 días de edad. El diseño experimental fue completamente al azar, con cuatro tratamientos $(0,10,15$ y 20\% de harina de hojas de morera; T1, T2, T3 y T4, respectivamente), cada uno con cuatro repeticiones y 8 aves por jaula. El comportamiento del testigo (T1) y el T3, fue similar $(P>0.05)$ en cuanto a porcentaje de postura, consumo de alimento g/ave/día, conversión kg de alimento/kg de huevos producidos y conversión $\mathrm{kg}$ de alimento/docena de huevos producidos. Los cuatro tratamientos fueron diferentes en cuanto al peso de los huevos $(P<0.001)$. Respecto a la eficiencia proteica, la utilización del $20 \%$ de harina de hojas de morera $(\mathrm{HHM})$ mostró menores rendimientos respecto de los otros tratamientos $(\mathrm{P}<0.001)$; el valor biológico de las proteínas fue similar para los cuatro tratamientos $(P>0.05)$. La mejor rentabilidad económica, relación beneficio/costo y precio por kilogramo de ración, fueron las del T3, seguidas del T1, T2 y por último el T4. Los resultados productivos, nutricionales y económicos, encontrados en este trabajo, permiten concluir que el mejor de los tratamientos, es el que contiene $15 \%$ de harina de hojas de morera (Morus alba) en reemplazo del 
concentrado comercial, y que no es recomendable llegar hasta un nivel de reemplazo del $20 \%$, porque la producción disminuye.

Palabras clave: Aves, alimentación, árboles forrajeros.

\section{ABSTRACT}

This work was made with the objective to evaluate the productive, nutritional and economic behavior in laying Japanese quails (Coturnix coturnix japonica) of week 2 to 23 of laying, using meal of leaf of mulberry (Morus alba) in three leaves the substitution the commercial concentrated. 128 birds of 52 days of age were used. Completely randomized design, with four treatments $(0,10,15$ and $20 \%$ of meal of leaf of mulberry T1, T2, T3 and T4, respectively), each one with four repetitions and 8 birds by cage. Was not significant difference between the witness (T1) and the T3, in percentage of posture, food intake $\mathrm{g} / \mathrm{bird} /$ day, conversion $\mathrm{kg}$ feed $/ \mathrm{kg}$ eggs produced and conversion $\mathrm{kg}$ feed/dozen eggs produced. ( $>>0.05)$, It was observed significant difference of the weight of eggs $(P<0.001)$ between the four treatments. The biological test for protein efficiency, showed to significant differences $(P<0.001)$ between the T4 and the other three treatments, as far as the biological value of proteins was not significant difference $(P>0.05)$. The best economic yield, relation benefit/cost and price by kilogram of diet, were the reported ones for the T3, following of the T1, T2 and finally the T4. The productive, nutritional and economic results, found in this work, allow to conclude that the best treatment is the one that contains $15 \%$ of mulberry leaf flour (Morus alba) in replacement of the commercial concentrated, and that is not recommendable to arrive available until a level of $20 \%$, because the production diminishes.

Keywords: Birds, feeding, fodder trees.

\section{INTRODUCCIÓN}

En Colombia no ha existido tradición de consumo de huevo y carne de codorniz, pero durante los últimos quince años ha surgido dentro de la actividad avícola, como una rama de extraordinario interés la cotornicultura. Esta actividad presenta 
ventajas importantes; como el requerimiento de un reducido capital, poco espacio para las instalaciones y bajos costos de mano de obra que favorecen su explotación. Por esto la explotación de codornices se ha desarrollado hasta el momento principalmente como una microempresa, por tal razón la información sobre producción en este reglón de la avicultura es insuficiente y restringida. La codorniz ha demostrado alta precocidad y productividad en áreas de clima templado y cálido de nuestro país, esto la hace una especie promisoria para su explotación comercial, por esta razón se hace necesario realizar estudios sobre esta especie, principalmente en materia de alimentación, pues ésta representa gran parte de los costos de producción.

En este estudio, se propone utilizar la harina de hojas de morera en reemplazo de un porcentaje del concentrado comercial para la alimentación de codornices en postura, sin que se disminuya la producción de huevo.

\section{MATERIALES Y MÉTODOS}

Se utilizaron 128 codornices de la raza japónica (Coturnix coturnix japonica) de 40 días de nacidas, en un diseño completamente al azar las aves fueron distribuidas en cuatro tratamientos; cada uno formado por cuatro repeticiones y ocho aves por repetición. Aunque desde el primer día las aves aceptaron el alimento, se les suministro la dieta correspondiente durante los diez primeros días como periodo de adaptación, al término de los cuales se inició la correspondiente toma de datos, el periodo experimental fue de cinco meses, Los tratamientos se distribuyeron así: $100 \%$ concentrado comercial codornices en postura (control), $90 \%$ concentrado comercial $+10 \%$ harina de hojas de morera, $85 \%$ concentrado comercial $+15 \%$ harina de hojas de morera y $80 \%$ concentrado comercial $+20 \%$ harina de hojas de morera, tratamientos $1,2,3$ y 4 respectivamente. La composición nutricional (Tabla1) de las dietas experimentales, se realizaron en el Laboratorio de Nutrición Animal.

Los parámetros evaluados fueron: porcentaje de postura, peso de los huevos, consumo de ración, conversión alimenticia $\mathrm{kg} / \mathrm{kg}$ y $\mathrm{kg} /$ docena de huevos, eficiencia 
proteica y valor biológico de la proteína, también se evalúo los costos de producción, rentabilidad y relación beneficio/costo de los tratamientos.

\section{RESULTADOS Y DISCUSIÓN}

Los resultados para el porcentaje de postura (Tabla 2 y Figura 1) son similares para los tratamientos 1,2 y 3 ( $P>0.05)$; el T1 con un $78.21 \%$ de postura, mostró una marcada diferencia en comparación con el T4 $69.60 \%(P<0.05)$, esto indica que el nivel más alto de reemplazo de HHM por concentrado comercial utilizado en este trabajo afectó negativamente la producción de huevos, sin embargo los tratamientos T3 y T4 fueron similares $(\mathrm{P}<0.01)$ con un 78.92 y $78.21 \%$ de postura respectivamente; (Figura 1). Los resultados no concuerdan con los encontrados por Marín y Encizo, (2003) con un 54-60\%; y de los reportados por Gamboa y Díaz, (2003) con 59.37-69.30\% utilizando soya integral tostada y soya integral cocida, en reemplazo de torta de soya como proteína vegetal respectivamente. Sin embargo, los resultados de este trabajo son más cercanos a Murakami et al., (1993) quienes reportan porcentajes de postura entre 79.42 y $81.93 \%$ y a los conseguidos por Vandepopulire, (1995) con 60-82\% de postura.

Tabla 1. Composición nutricional de los tratamientos, porcentajes en materia seca.

\begin{tabular}{lccccc}
\hline \multicolumn{1}{c}{ Elemento } & $\boldsymbol{H . H . M}$ & $\begin{array}{c}\text { T1 } \\
\text { C.C }\end{array}$ & $\begin{array}{c}\text { T2 } \\
\mathbf{1 0 \%} \\
\text { morera }\end{array}$ & $\begin{array}{c}\text { T3 } \\
\mathbf{1 5 \%} \\
\text { morera }\end{array}$ & $\begin{array}{c}\text { T4 } \\
\mathbf{2 0 \%} \text { morera }\end{array}$ \\
\hline Proteína & 18.46 & 19.43 & 18.36 & 18.57 & 18.63 \\
Grasas & 1.16 & 5.29 & 5.22 & 4.13 & 3.57 \\
Fibra Cr. & 13.55 & 4.76 & 4.49 & 6.42 & 4.68 \\
Cenizas & 7.7 & 9.76 & 10.97 & 9.64 & 9.13 \\
ENN & 56.92 & 56.32 & 56.62 & 56.68 & 60.37 \\
NDT & 74.53 & 78.19 & 77.07 & 76.35 & 79.25 \\
E.D Mcal/kg & 3.29 & 3.45 & 3.40 & 3.37 & 3.49 \\
E.M Kcal/kg & 2690 & 2830 & 2790 & 2760 & 2870 \\
Relac. E/P & 145.72 & 145.65 & 151.96 & 148.62 & 154.06 \\
\hline Fuen $/$ Labon
\end{tabular}

Fuente: Laboratorio de Nutrición Animal Universidad de los Llanos, 2003.

$\mathrm{HHM}=$ Harina de hojas de morera. C.C = Concentrado comercial. 
El peso de los huevos es notablemente diferente entre los cuatro tratamientos $(\mathrm{P}<0.001)$ (Tabla 2); el tratamiento con el $20 \%$ de $\mathrm{HHM}$ registra como peso promedio de los huevos $10.48 \mathrm{~g}$, siendo el de mejores resultados, seguido por T1 $10.19 \mathrm{~g}, \mathrm{~T} 210.06 \mathrm{~g}$ y por último por T3 con $9.92 \mathrm{~g}$, el cual tiene un peso promedio más bajo en este estudio (Figura 2). Lo anterior no coincide con lo encontrado por Marín y Encizo, (2003) con pesos de 10.4-10.7 g ni por Gamboa y Díaz, (2003) con 10.21-10.63 g; lo estudiado en este trabajo es muy cercano a los resultados de Murakami et al., (1993) quienes trabajaron con niveles de energía y proteína cercanos a los encontrados en este estudio, reportan huevos con pesos de 9.96$10.48 \mathrm{~g}$.

El reemplazo de concentrado comercial por HHM produjo aumento en el consumo de las raciones experimentales respecto a la testigo $(P<0.001)$; la Tabla 2 muestra estas diferencias; el consumo más alto es el reportado para las aves alimentadas con el T4 con $26.64 \mathrm{~g}$, seguido en forma descendente por T2 con $25.38 \mathrm{~g}$ y por último por T3 con $24.85 \mathrm{~g}$, este último es similar al testigo $(P>0.05)$ el cual presentó el consumo más bajo $24.78 \mathrm{~g}$. A pesar de que los niveles de energía metabolizable (EM) reportados por el laboratorio (Tabla 1), son altos para el tratamiento del $20 \%$ de HHM, éste presenta los consumos más altos, siendo este comportamiento contrario a lo esperado; pues según McDonald, (1999) las gallinas ponedoras ajustan el consumo de pienso de acuerdo a si hay una alta concentración energética en la ración; el mismo autor aclara que la composición de raciones mixtas, es siempre menor de la esperada y más notable si se mezcla concentrados con alimentos groseros. Los tratamientos 1, 2 y 3 presentan un alto contenido de extracto no nitrogenado y una menor proporción de los principales componentes de la dieta (proteína, grasa, fibra y cenizas); el aumento de los carbohidratos en el T4 explica el alto contenido de energía metabolizable, esto también puede explicar el mayor consumo de ración de las codornices alimentadas con el T4 con la posibilidad de que gran parte del extracto no nitrogenado esté formado por carbohidratos poco digeribles para las codornices, lo cual produjo un reporte de altos niveles de energía por el laboratorio. El consumo de alimento ave/día encontrado en este trabajo coincide con lo reportado por 
Murakami et al., (1993) con consumos de 25.50-26.39 g, también con lo reportado por Vandepopuliere, (1995) 23.5-27.4 g; no coincide con Marín y Encizo, (2003) ni Gamboa y Díaz, (2003) pues en esos estudios los consumos fueron menores, 23.8-24.9 g y 22.85-24.71 g, respectivamente.

La mejor conversión la mostró T1 con $3.08 \mathrm{~kg} / \mathrm{kg}$, seguido del T3 con 3.17, considerando este comportamiento similar $(P>0.05)$, en las aves alimentadas con estas raciones; el testigo fue mejor al T2 cuya conversión fue 3.36, $(P<0.05)$; el peor valor fue 3.64 para el T4, este tratamiento presento una conversión muy lejana a la reportada para el testigo $(P>0.001)$ y un comportamiento diferente con T2 $(P<0.05)$ y con el T3 $(P<0.01)$, entre el T2 y T3 no se encontró diferencia significativa $(P>0.05)$. Los resultados de este trabajo (Tabla 2 ) son cercanos a los reportados por Murakami et al., (1993) con 3.00-3.33; y difieren de los reportados por Marín y Encizo, (2003) 3.45-3.87, y de los mostrados por Gamboa y Díaz, (2003) con 3.52-4.33.

Tabla 2. Desempeño de Codornices Alimentadas con Tres Niveles de Harina de Hojas de Morera en Reemplazo del Concentrado Comercial, Entre las Semanas 2 a 23 de Postura.

\begin{tabular}{lcccc}
\hline \multicolumn{1}{c}{ VARIABLES } & T1 & T2 & T3 & T4 \\
\hline Porcentaje de postura (ave/día) & $78.21^{\mathrm{a}}$ & $75.45^{\mathrm{ab}}$ & $78.92^{\mathrm{a}}$ & $69.60^{\mathrm{b}}$ \\
Peso huevos g/ave/día. & $10.19^{\mathrm{b}}$ & $10.06^{\mathrm{c}}$ & $9.92^{\mathrm{d}}$ & $10.48^{\mathrm{a}}$ \\
Consumo de ración g/ave/día. & $24.78^{\mathrm{c}}$ & $25.38^{\mathrm{b}}$ & $24.85^{\mathrm{bc}}$ & $26.64^{\mathrm{a}}$ \\
Conv Alim (kg ración/kg huevos). & $3.08^{\mathrm{c}}$ & $3.36^{\mathrm{b}}$ & $3.17^{\mathrm{bc}}$ & $3.64^{\mathrm{a}}$ \\
Conv Alim (kg /docena de huevos). & $0.38^{\mathrm{c}}$ & $0.41^{\mathrm{b}}$ & $0.38^{\mathrm{c}}$ & $0.46^{\mathrm{a}}$ \\
Eficiencia proteica. & $6.09^{\mathrm{b}}$ & $6.15^{\mathrm{b}}$ & $5.84^{\mathrm{b}}$ & $7.15^{\mathrm{a}}$ \\
Valor biológico de la proteína (\%) & 73.66 & 74.63 & 74.81 & 68.31 \\
\hline
\end{tabular}

Letras distintas entre columnas indican diferencias significativas entre tratamientos en la prueba de Tukey $(\mathrm{P}<0.05)$.

En cuanto a la conversión $\mathrm{kg} /$ docena de huevos, fueron $(P>0.05)$ los tratamientos 1 y 3 , los que presentaron una conversión de 0.38 , siendo este el mejor valor promedio para este parámetro en tiempo de trabajo (Tabla 2 y Figura 3 ), estos dos tratamientos fueron superiores al T2 $(\mathrm{P}<0.05)$, siendo la conversión para este de 
0.41, seguida por la del T4 con $0.46(\mathrm{P}<0.001)$. La conversión alimenticia $\mathrm{kg} /$ docena de huevos, brinda la medida efectiva para evaluar el comportamiento productivo de codornices en la etapa de postura, pues en este tipo de producción no se clasifica los huevos según el tamaño; lo importante es la cantidad de huevos producidos. Lo hallado en el presente trabajo difiere de lo reportado por Marín y Encizo, (2003) con conversiones de 0.41-0,50 y de lo encontrado por Gamboa y Díaz, (2003) con valores de 0.44-0.47; es cercano a lo reportado por Murakami et al., (1993) con valores de 0.38-0.39 de conversión.

La eficiencia proteica de los tratamientos 1,2 y 3 (Tabla 2$)$, fue similar $(P>0.05)$, el T4 fue superior $(\mathrm{P}<0.001)$ con relación a los otros tratamientos. Para el T1 se encontró que las codornices alimentadas con el concentrado comercial utilizado como testigo en el presente trabajo, con 19.43\% de proteína bruta, necesitan digerir $6.09 \mathrm{~g}$ de esta proteína diariamente, es decir con ese nivel de proteína y un manejo adecuado, las aves de este tratamiento no llegarían a producir en forma constante más de un $85 \%$ de huevos, esto con las condiciones y promedios de postura que hubo en los cinco días en que se midió este parámetro. Las aves alimentadas con la ración de $18.36 \%$ de proteína bruta, en la cual se ha reemplazado un $10 \%$ del concentrado comercial por harina de hojas de morera, necesitan de $6.16 \mathrm{~g}$ de esta proteína para producir de forma normal hasta un $81 \%$ de huevos. Las codornices alimentadas con una ración en la cual se reemplazó un 15\% del CC por HHM y que contiene un 18.57\% de proteína bruta, necesitan 5.84 g de esta proteína para producir hasta un $83 \%$ de huevos en forma constante. Las codornices alimentadas con raciones en las cuales se ha reemplazado un $20 \%$ del CC por HHM y que contienen $18.63 \%$ de proteína bruta, necesitan consumir 7.15 g de esta proteína para producir en forma regular hasta un $74 \%$ de huevos. Para este parámetro no se encontró ningún trabajo similar, solo se puede citar lo expresado por Bissoni, (1996) "la codorniz ponedora, necesita consumir como mínimo $5.29 \mathrm{~g}$ de proteína diariamente"; respecto a esto, lo encontrado en este trabajo es diferente, pues el valor más cercano es de $5.84 \mathrm{~g}$ de proteína diaria, se dio en las aves alimentadas con 15\% de HHM; para los otros tratamientos los valores de proteína consumida son más altos. 


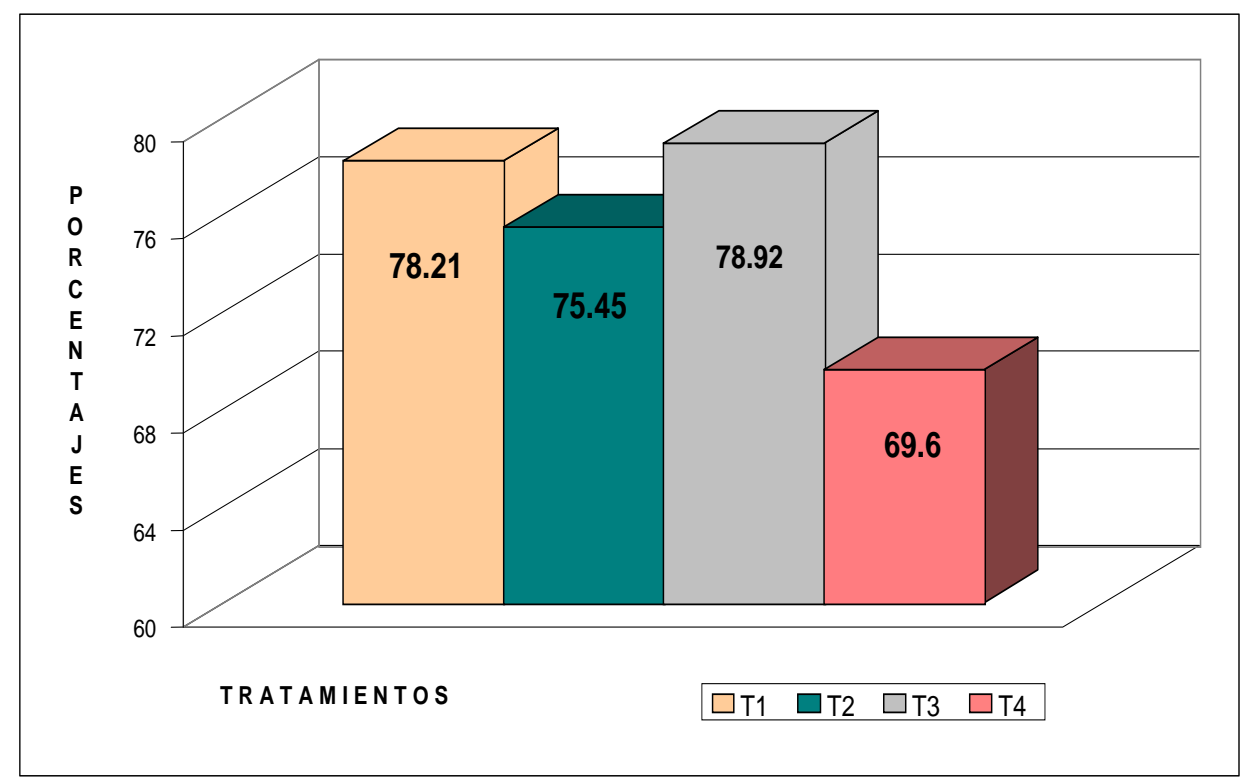

Figura 1. Porcentaje de postura en los cuatro tratamientos

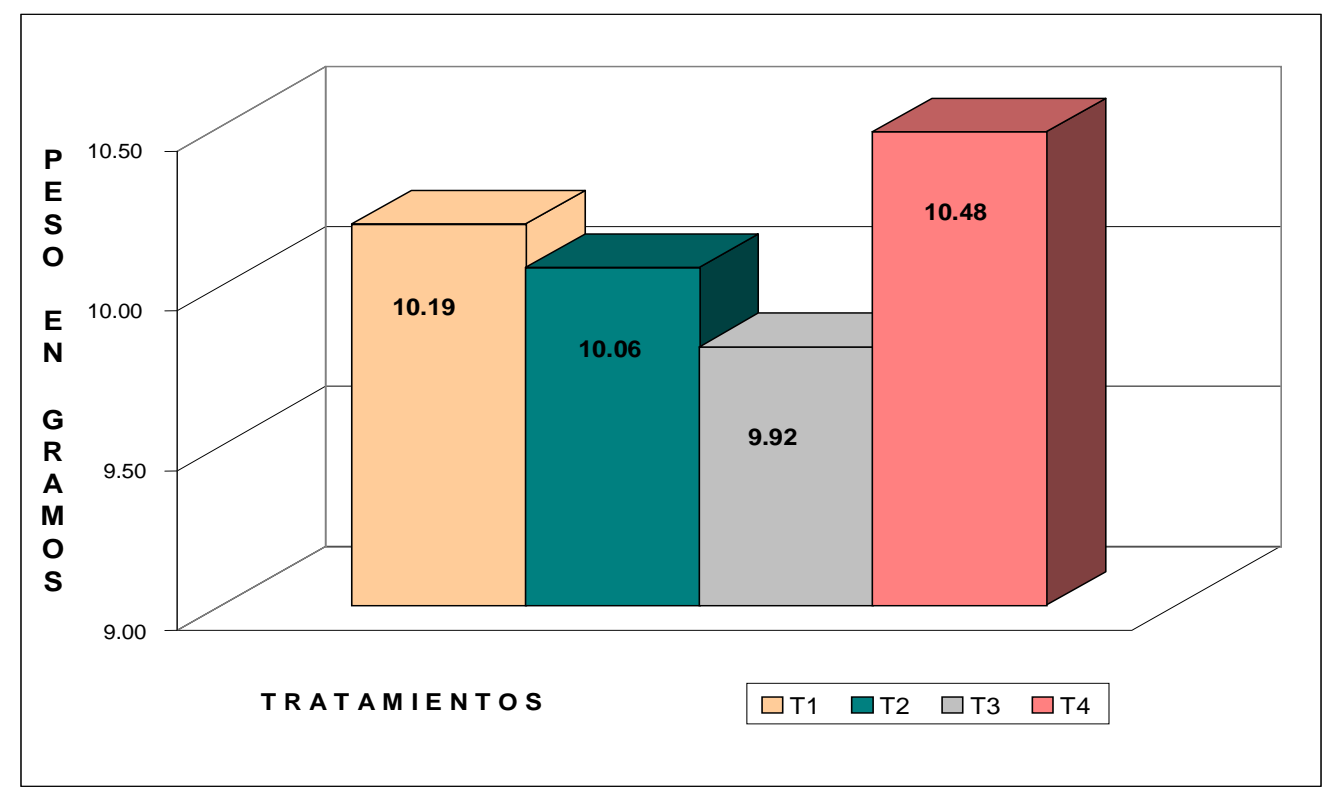

Figura 2. Peso promedio de los huevos en los cuatro tratamientos

El valor biológico de la proteína fue similar $(P>0.05)$, en los cuatro tratamientos, (Tabla 2); sin embargo, se define que el mejor valor se dio para el T3 con $74.81 \%$, esta ración fue $1.15 \%$ más absorbida que la de $\mathrm{T} 1$, en este trabajo la mejor proteína fue la de las raciones en las cuales se reemplazó un 15\% del CC por 
HHM, seguido por el T2 con $74.63 \%$, el T1 $73.66 \%$ y por último el T4 con $68.31 \%$ de valor biológico de su proteína. No se encontró trabajos similares o en los cuales se midiera esta variable en aves ponedoras.

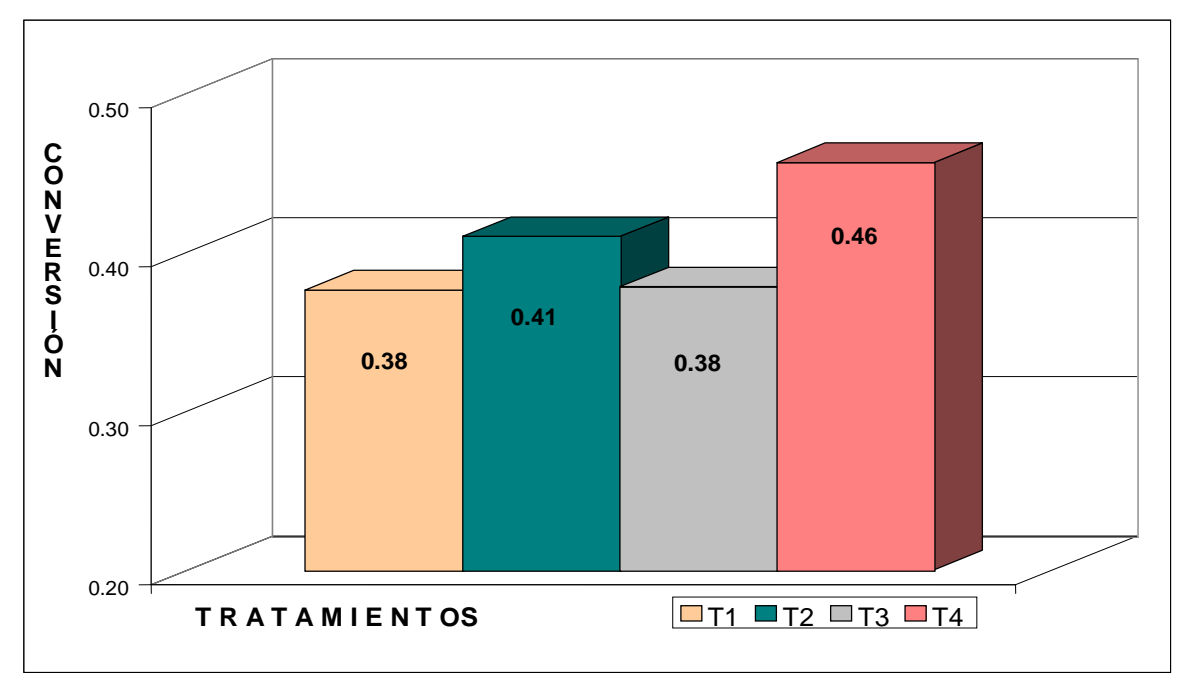

Figura 3. Peso promedio de los huevos en los cuatro tratamientos

Al igual que en los trabajos desarrollados por Naraya y Setty, (1997); Uchino et al., (1988); y Suda, (1999) en los cuales se ofreció hojas de morera como alimento a aves ponedoras; en este trabajo también se encontró que la inclusión de las hojas de esta planta en la alimentación de las aves, mejora la coloración de la yema, e incluso de la cascara de los huevos de codorniz.

\section{CONCLUSIONES}

El reemplazo de concentrado comercial por harina de hojas de morera (Morus Alba) en la alimentación de codornices ponedoras, no afecta la producción de huevos cuando este reemplazo no supera el $15 \%$ de la ración, incluso este nivel da resultados un tanto mejores que los conseguidos con concentrados de las características químicas del utilizado en este estudio; sin que la diferencia sea significativa. El uso de harina de hojas de morera en la alimentación de codornices ponedoras no afecta el peso comercial de los huevos. Con el reemplazo de $10 \mathrm{y}$ $20 \%$ del concentrado comercial por harina de hojas de morera se presenta un aumento en el consumo de ración. El reemplazar un 15\% del concentrado 
comercial por HHM, no afecta la conversión alimenticia $\mathrm{kg} /$ docena de huevos. En cuanto a la de eficiencia proteica, los mejores resultados fueron para el T3; aunque no se halló diferencia significativa entre este y el T1, las aves del tratamiento con $15 \%$ de HHM necesitan consumir menos proteína para obtener una buena producción de huevos. Es más importante la relación energía/proteína que el valor de cada uno de estos elementos por separado en el alimento, pues esta relación en el concentrado y el T3, es más cercana a la recomendada y la del T4 es la más lejana.

Se recomienda utilizar harina de hojas de morera (Morus Alba) en niveles de hasta $15 \%$ en reemplazo del concentrado comercial. Elaborar dietas completas; isoproteicas e isocalóricas, con niveles de harina de hojas de morera del $5-20 \%$ del total de la dieta. Para que el presente trabajo sea tenido en cuenta como una alternativa real en la alimentación de codornices, es necesario replicarlo en condiciones de una explotación comercial y tener en cuenta todas las variables de carácter técnico, biológico, de manejo y sanidad que intervienen en este tipo de producción a escala industrial. Realizar estudios en los que se mida la coloración de la yema de los huevos, cuando se utiliza hojas de morera como parte del alimento.

\section{REFERENCIAS BIBLIOGRÁFICAS}

1. Bissoni E. Cría de la Codorniz. Argentina: Editorial Albatros, 120 p. 1996.

2. Elangovan A. V., Verma V. S., Sastry R. B., Singh S. D. Laying performance of Japanese quail fed graded levels of neem (Azadirachta indica) kernel meal incorporate diets. Animal Feed Science and Tecnology, 88: 113-120. 2000.

3. Gamboa O., Díaz, J. Efecto de la soya integral cocida en la alimentación de la codorniz (Coturnix cotuurnix japonica) sobre la producción de huevo y la calidad de la cáscara. Trabajo de grado (Veterinaria y Zootecnia). Universidad de los Llanos. Facultad de Ciencias Agropecuarias y recursos Naturales. Villavicencio, 52 p. 2003.

4. Gonzales A. G. Fundamentos de Nutrición Animal Aplicada. Colombia: Editorial Universidad de Antioquia, p 62-64, 2001.

5. Gutiérrez R., Roa M. L. Determinación de algunos compuestos químicos en cuatro plantas arbóreas forrajeras. Revista Colombiana de Ciencias Pecuarias. Universidad de Antioquía, 16 (2), 2003.

6. Institut National de la Recherche Agronomique. L'alimentation Des Animaux Monogastrigues, París, 282 p. 1988. 
7. Lee W. C., Kim S. Potencial de hojas de morera para el control de enfermedades que atacan población senil. Simposio Conmemorativo para el 40ํㅡㄹ Aniversario de la Sociedad Coreana de Ciencia Serícola, Seúl. p 121. 1997.

8. Marín I., Enciso M. Efecto de la soya integral tostada en la alimentación de la codorniz (Coturnix cotuurnix japonica) sobre la producción de huevo y la calidad de la cáscara. Trabajo de grado (Veterinaria y Zootecnia). Universidad de los Llanos. Facultad de Ciencias Agropecuarias y Recursos Naturales. Villavicencio, 54 p. 2003.

9. McDonald P., Edwards R. A., Greenhalgh J. F., Morgan C. A. Animal Nutrition. Traducido por Arias, S. R. Nutrición Animal. 5 ${ }^{a}$ ed, Acribia S.A., España, p. 215-278. 1999.

10. Murakami A. E., Barbosa de M. V. M., Ariki J., Junqueira O. M., Kronka, S. Níveis de proteína e energia em rações para codornas japonesas (Coturnix coturnix jáponica) em postura. Revista Brasilera de Zootecnia, 22 (4): 541-551, 1993.

11. Narayana H., Setty S. Studies on the incorporation of mulberry leaves (Morus indica) in layers mash on health, production and egg quality. Indian Journal of Animal Science, 47 (4): 212-215. 1977.

12. National Research Council. Committee on animal nutrition. Subcommittee on poultry nutrition. Nutrient requirements of poultry. $9^{\text {th }}$ ed. Washington: National Academy of Sciences, 155 p. 1994.

13. Parada F. J. A., Serna, P. M. Utilización de la harina de lombriz (Eisenia foétida) en la alimentación de codornices (Coturnix coturnix japónica) en postura. Trabajo de grado (Zootecnista). Universidad Nacional de Colombia. Facultad de Medicina Veterinaria y Zootecnia. Bogotá, 58 p. 1994.

14. Romero O., Bolaños P. Ensayo de alimentación con harina de morera en codornices en postura. Universidad de los Llanos, 12 p. 1998.

15. Sánchez M. D., Rosales M. Agroforestería para la producción animal en América Latina. Memorias de la conferencia electrónica realizada de abril a septiembre de 1998. Estudio FAO Producción y Sanidad Animal, n.143, Roma. 515 p. 1999.

16. Santacoloma V. L. Nutrición de monogástricos. Bogotá: UNAD, Facultad de Ciencias Agropecuarias, 215 p. 1998.

17. Shayo C. M. Uses, yield and nutritive value of mulberry (Morus alba) trees for ruminants in the semi-arid areas of central Tanzania. Tropical Grasslands, 31 (6): 599-604. 1997.

18. Silva B. M. T., Cotta J. T., Oliveira A. I. Níveis de energia metabolizável em rações de codornas japonesas (Coturnix coturnix jáponica) na fase inicial de postura. Ciência y agrotecnología, Lavras, 24 (3): 782-793. 2000.

19. Singh B., Goel G. C., Negi, S. Effect of supplementing muberry (Morus alba) leaves ad libitum to concentrate diets of Angora rabbits on wool production. Journal of Applied Rabbit Research, 7 (4): 156-160. 1984.

20. Suda T. Inhibitory effect of mulberry leaves on ammonium emission from poultry excrement. Abstracts of Gunma Agriculture-related Experiment Stations Meeting, 7: 8. 1999. 


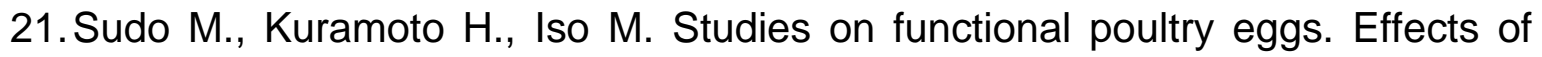
mulberry leaves on quantity and quality of eggs. Bulletin of Ibaraki Prefectural Poultry Experiment Station, 35: 15-17, 2000.

22. Trigueros R. O., Villalta, P. Evaluación del uso de follaje deshidratado de morera (Morus alba) en alimentación de cerdos de la raza Landrace en etapa de engorde. CENTA, El Salvador. p 150-155. 1997.

23. Uchino K., Watanabe M., Ishida H., Sato A. Effect of guinea fowls raised in a mulberry garden. Bulletin of Chiba Prefectural Sericulture Experiment Station, 6: 1-10. 1988.

24. Vandepopulire J. M. Al-Yousef, Y. Lyons, J.J. Dates and date pits as ingredients in broiler starting and coturnix quail breeder diets. Poultry Science, 74 (7): 1134- 1142. 1995.

25. Zhuang H. Fisiología del Gusano de Seda. Seúl, p 50, 1998. 